\title{
Comparison of general anesthesia and combined spinal and epidural anesthesia for gasless laparoscopic surgery in gynecologic field
}

\author{
Jong Ha Hwang ${ }^{1}$ and Bo Wook Kim ${ }^{1}$ \\ ${ }^{1}$ Affiliation not available
}

July 7,2020

\begin{abstract}
Objective: This study aimed to compared general anesthesia and combined spinal and epidural anesthesia (CSEA) for gasless laparoscopic surgery in gynecologic field. Study design: We matched patients with type of surgery who underwent gasless single port access (SPA) laparoscopic surgery under general anesthesia (GA) and CSEA. The medical records of 90 patients between March 2018 and June 2020 were reviewed. Gasless laparoscopic surgery was performed in all patients with a single-port access (SPA) using a J-shaped retractor Results: No significant differences were observed for age, body mass index, parity, and previous abdominal surgery between GA and CSEA group. During operation under CSEA, six patients (20\%) experienced nausea/vomiting. Hypotension (systolic blood pressure $<90 \mathrm{mmHg}$ ) was observed in five patients $(16.7 \%$ ). Intravenous analgesics was administrated in four of the patients $(13.3 \%)$ who suffered from shoulder pain or abdominal discomfort. One patient developed bradycardia. The duration of hospital admission was shorter in the CSEA group than in the GA group (p value $=0.014)$. There was no difference between the groups in terms of surgery type, surgical specific finding, operation time, estimated blood loss, laparotomy conversion rate and use of additional trocar. No major complications such as urologic, bowel, or vessel injuries were found in both groups. Conclusions: CSEA is a safe and feasible technique for application in non-obese patients undergoing gasless laparoscopic surgery in gynecologic field.
\end{abstract}

\section{INTRODUCTION}

General anesthesia (GA) is the preferred and predominant technique for laparoscopic surgeries such as adnexal surgery, total hysterectomy and myomectomy in gynecologic field because it controls surgical pain and improves patient comfort with pneumoperitoneum and Trendelenburg position. General anesthesia provides a secure airway and allows for the precise control of ventilation to reduce hypercarbia $(1,2)$.

The benefits of regional anesthesia, such as combined spinal and epidural anesthesia (CSEA), include reduction in the side effects of general anesthetics such as nausea, vomiting, sore throat, dental injury, sedation, postoperative atelectasis, and hypoventilation $(3,4)$. Sequelae of general anesthesia such as airway trauma, myalgia, and sore throat can be avoided with regional anesthesia. The later also allows for earlier cognitive recovery and oral intake in the immediate postoperative period, whereas its long-term benefits have not yet been clearly demonstrated. Additional potential benefits of regional anesthesia include rapid recovery, effective postoperative analgesia and early ambulation and recover. Regional anesthesia has been used in laparotomy for patients with poor cardiopulmonary function because of low burden on cardiopulmonary function. However, the implementation of laparoscopic surgery under regional anesthesia has been limited. Regional anesthesia may be associated with adverse effects such as severe hypotension, shoulder discomfort due to diaphragmatic irritation and respiratory discomfort by pneumoperitoneum.

Most reports of regional anesthesia in laparoscopic surgery involved cholecystectomy and few cases of appendectomy (5-7). Laparoscopic cholecystectomy is performed in a reverse Trendelenburg position, which results in a more favorable cardiopulmonary function. The operation time of laparoscopic appendectomy 
is relatively short. Therefore, regional anesthesia could not be applied to gynecologic laparoscopic surgery, which is performed in the Trendelenburg position. Trendelenburg position under regional anesthesia worsens pulmonary function and induces hypercarbia owing to hypoventilation together with carbon dioxide $\left(\mathrm{CO}_{2}\right)$ pneumoperitoneum in laparoscopic surgery. Regional anesthesia used in combination with gasless laparoscopy can be an alternative to overcome the limitation of performing gynecologic minimal surgery in the Trendelenburg position. Here, we evaluated the feasibility and efficacy of gasless laparoscopic surgery including laparoscopic hysterectomy, laparoscopic myomectomy, laparoscopic adnexal surgery in gynecologic field under CSEA.

\section{METHODS AND METHODS}

\section{Study design and patient selection}

The study was reviewed and approved by the institutional review board of International St. Mary's Hospital, Catholic Kwandong University College of Medicine, Incheon, Republic of Korea (IS19RISI0043). The medical records of patients who underwent gasless single port access (SPA) laparoscopic surgery under CSEA or GA at the Catholic Kwandong University, International Saint Mary's Hospital, Incheon, South Korea, between March 2018 and June 2020 were reviewed. We matched a 1:2 cohort of patients by surgery type who underwent gasless laparoscopy in gynecologic field. All patients provided informed consent to undergo laparoscopic hysterectomy and conversion to laparotomy if needed. After being counseled by the surgeon about the advantages and risks of undergoing general and CSEA, the patients chose the anesthesia method during their preoperative visit. The patients were explained that CSEA will be converted to GA when CSEA is found insufficient or when patients expressed their wish to do so. The patients were encouraged to report any discomforts, such as abdominal pain, shoulder pain, nausea, and vomiting, experienced during the operation. They were also explained that any anxiety, pain, or discomfort occurring during surgery could be dealt with medications. The exclusion criteria included body mass index (BMI) of $>30$, infection at the site of injection, sepsis, uncorrected hypovolemia, increased intracranial pressure, lack of cooperation or patient refusal, neurologic diseases, coagulopathy, and allergy to local analgesics.

\section{Anesthetic procedure (CSEA)}

The same anesthesiologist (YS Choi) performed CSEA in all cases. The patients were positioned in the right lateral position. Lumbar area was draped and prepared. Lidocaine at a concentration of $2 \%$ was administrated intradermally for local anesthesia. L3-L4 interspace was palpated. Under strict aseptic precautions, a single puncture spinal and epidural block was administered using CSE set, and an 18-G needle was used for tunneling. Using the loss of resistance technique to saline technique, the $18-\mathrm{G}$ Touhy needle was inserted into the epidural space at midline approach. The $26-$ or $27-\mathrm{G}$ pencil point spinal needle was advanced into the intrathecal space through an epidural needle to determine the subarachnoid space. After the flow of cerebrospinal fluid was confirmed, $0.5 \%$ bupivacaine $8-10 \mathrm{mg}$ plus $10 \mathrm{mcg}$ fentanyl was injected into L3-L4 or L4-L5 subarachnoid space. Finally, the spinal needle was removed and epidural catheter was placed into the epidural space in the cephalic direction and fixed at $3-4 \mathrm{~cm}$ within the epidural space. The patients were turned to the supine position and were subjected to a $15^{\circ}-20^{\circ}$ Trendelenburg tilt for achieving the required level of block. Sensory block level was checked by performing a pinprick test. The target level was T4, and the surgery was initiated when the block reached the T4 level. When the operation was longer, $1 \%-2 \%$ lidocaine $(10-15 \mathrm{cc})$ was repeatedly injected via the epidural catheter.

\section{Surgical procedure}

All surgeries were performed by two laparoscopic surgeon (BW Kim, JH Hwang). In the operative room, intravenous (IV) lines were placed and all patients received adequate preloading with $1000 \mathrm{~mL}$ of Ringer's lactate solution. Patients were advised to cleanse their bowels the day before the operation. Patients were placed in a dorsal lithotomy position. Antibiotics (cefotetan $1 \mathrm{~g}$ ) were administered immediately prior to the surgery. Abdominal cleansing and sterile draping were performed. The umbilical region was cleansed with fine-tipped instruments such as hemostatic forceps. A RUMI system uterine manipulator (Cooper Surgical, Trumbull, CT, USA) was utilized with a balloon tip inserted into the uterine cavity after disinfection and 
sterile draping.

After making a vertical incision of $2-2.5 \mathrm{~cm}$ on the skin within the umbilicus, and the subcutaneous tissue was bluntly dissected using the tip of an instrument such as a Kelly clamp. The access route into the abdominal cavity was created by subsequent incisions of the fascia and peritoneum. The umbilical wound was expanded using an Alexis retractor (Applied Medical, Rancho Santa Margarita, CA, USA) or an Endo Keeper (Nelis, Bucheon-si, Gyeonggi-do, South Korea). For gasless laparoscopy, abdominal wall retraction was performed using a J-shaped retractor (DCT Korea, Seoul, South Korea). A J-shaped retractor was a surgical instrument that was designed for attachment to the Thompson surgical retractor. The process of abdominal wall retraction using the J-shaped retractor and attachment to the Thompson retractor is described in Figure 1. Abdominal suspension suture was not used in all patients. After completing abdominal wall retraction, a $30^{\circ}$ rigid laparoscope $(5 \mathrm{~mm})$ was inserted into the pelvic cavity via the umbilical incision. When an ancillary port was required, one trocar was introduced into the suprapubic area. Trendelenburg position was maintained to clear the bowel in the operative field. Procedures such as adhesiolysis, appendectomy, and myomectomy were performed as needed. The surgical procedures of total hysterectomy, myomectomy, adnexal cystectomy, salpingo-oophorectomy, and salpingectomy were performed according to standard protocols with no modification. After the surgical procedures were completed, the wound retractor was removed and the umbilical wound was closed.

\section{Patient monitoring and treatment of adverse effects}

All routine monitors for non-invasive blood pressure and heart rate measurements as well as pulse oximeter $\left(\mathrm{SpO}_{2}\right)$ were attached, and the baseline values of vital signs were recorded. To relieve patient anxiety during operation, the use of ear phones was allowed if required by the patients. Hypotension (systolic arterial pressure $<90 \mathrm{mmHg}$ ), bradycardia ( $\mathrm{HR}<50$ beats/min), hypoxemia $(\mathrm{SpO} 2<90 \%)$, headache, nausea/vomiting, shoulder pain, anxiety, and abdominal pain were recorded. Hypotension was treated with ephedrine or phenylephrine. Bradycardia was treated with IV administration of $0.5 \mathrm{mg}$ atropine, while nausea/vomiting was treated with IV metoclopramide or ondansetron. Anxiety was treated with IV midazolam, while shoulder pain or abdominal discomfort was treated with IV fentanyl or morphine.

\section{Data collection}

The demographic and physical characteristics of patients were obtained preoperatively. Data included age, height, BMI, parity, previous abdominal surgery, and tumor marker (CA 125) level. Total operative time, set up time, estimated blood loss, type of surgery, and concomitant surgery were recorded. Setup time was defined as the time from abdominal incision of the umbilicus to pathway construction into the abdominal cavity, application of wound retractor, and setting up of Thompson surgical retractor and J-shaped retractor. Major complications included urologic, bowel, and vessel injuries. Laparotomy conversion rate, use of additional trocar, and specific surgical findings (such as severe pelvic adhesion, mass rupture and torsion) were evaluated. Adverse effects during CSEA were also evaluated.

\section{RESULTS}

30 patients in the CSEA group and 60 patients in the GA group were identified during the study period. Table 1 shows the demographics and clinical characteristics of patients who underwent gasless SPA laparoscopy for two groups. The overall mean age (year) was 41.4 and 42.6 in the CSEA and GA group, respectively, which showed no significant difference $(p=0.660)$. There is also no significant difference in BMI, parity, or the number of previous abdominal surgeries between two groups. Previous cesarean section is the most common type of previous abdominal surgery.

The characteristics of CSEA are shown in Table 2. Epidural catheter could be easily introduced in all the patients, and there were no conversions to GA. CSE block was performed at L3-L4 interspace in 29 (96.7 $\%)$ patients and at L4-L5 interspace in one $(3.3 \%)$ patient. Peak block height reached up to the T2 level. Peak block height was T4 level in 24 patients $(80 \%)$. During operation, lidocaine with or without $0.5 \mathrm{mg}$ morphine was injected through the epidural catheter in six patients $(20 \%)$. All patients remained conscious 
throughout the operation without respiratory depression and did not complain of any dyspnea. None of the patients showed an elevation $\mathrm{ETCO}_{2}$ level by $>20 \%$ from baseline. Midazolam $(1 \mathrm{mg})$ was administrated in 12 patients $(40 \%)$ to relieve anxiety. Nausea/vomiting requiring anti-emetics occurred in six patients (20\%). Hypotension was observed in five patients (16.7\%). IV analgesics were administrated in four of the patients (13.3) who had shoulder pain or abdominal discomfort. Bradycardia occurred in one patient, which was treated with a single dose of atropine $0.5 \mathrm{mg}$. No patients were shifted to general anesthesia owing to adverse effects.

Surgical outcomes are shown in Table 3. The most common type (54 cases, 60\%) of SPA laparoscopic surgery was adnexal surgery, followed by total hysterectomy (12 case, 20\%), and myomectomy (12 cases, 20\%) in both groups. There is no significant difference in specific surgical findings such as severe pelvic adhesion, mass rupture, and torsion between the two groups. The mean setup time from umbilical skin incision to abdominal wall retraction was lower in the CSEA group $(6.8 \mathrm{~min}$ ) than in the GA group $(7.7 \mathrm{~min})$ ( $p$ value $=0.012$ ). The mean hospital stay was shorter in the CSEA group (4.5 days) than in the GA group (5.0 day) $(p$ value $=0.014)$. The mean operation time was $68.9 \mathrm{~min}$ in the CSEA group and $78.3 \mathrm{~min}$ in the GA group, respectively $(p$ value $=0.210$ ). There is no significant difference in estimated blood loss between the two groups $(p$ value $=0.146)$.

There was two cases $(2.2 \%)$ of conversion to laparotomy, which involved severe pelvic adhesion. All gasless SPA laparoscopies, except three cases, were performed without an additional ancillary port insertion. No serious complications including urologic, bowel, or vessel injuries were noted in both the groups. There was no case of retraction-site abdominal wall injury.

\section{DISCUSSION}

Laparoscopic surgery is currently accepted as a feasible and effective way to treat gynecologic disease. GA has been regarded as a suitable anesthetic technique for laparoscopic surgery owing to the various effects of pneumoperitoneum (1). There are various complications associated with GA. Sympathetic stimulation caused by stress during intubation in addition to sympathetic activity occurring owing to pneumoperitoneum result in hypertensive episodes in patients. There is always a risk of unexpected difficult airway and aspiration of gastric contents.

Regional anesthesia such CSEA has been documented to be equally favorable in laparoscopic surgeries. Regional anesthesia is advantageous over GA in terms of overall safety, shorter postoperative stay, lesser emesis, lesser postoperative pain, and absence of airway manipulation (3). It leads to a faster recovery in immediate postoperative settings. Nausea and vomiting after GA administered using an inhalational anesthetic can be minimized. Regional anesthesia prevents the deterioration of respiratory system and facilitates early ambulation. It can be safely used in older patients with poor cardiopulmonary function and can be considered for used in emergency operation of patients who do not maintain a fast. If there is a fear of a serious side effect resulting from GA or a previously administered GA, regional anesthesia should be considered first.

Although, regional anesthesia has advantages over GA, laparoscopy has been performed under GA. Traditionally, $\mathrm{CO}_{2}$ pneumoperitoneum and Trendelenburg position were required to maintain the vision of operative field in laparoscopic gynecologic surgery, which could induce the risk of aspiration, $\mathrm{CO}_{2}$ gas load, nausea/vomiting, shoulder pain and decreased cardiopulmonary function. When regional anesthesia is applied during conventional laparoscopy using $\mathrm{CO} 2$ gas, nausea/vomiting and shoulder pain is main problem to patients. Especially, shoulder pain, which is referred to as the pain resulting from diaphragm stretching owing to insufflating $\mathrm{CO}_{2}$, is usual and intolerable events to patients under regional anesthesia (8). The diaphragm was innervated by cervical roots which are not covered during regional anesthesia. Intolerable shoulder pain during surgery is a major reason that GA is preferred to regional anesthesia. As a solution to shoulder pain, Sarli et al. suggested that the use of low-pressure pneumoperitoneum $(<10 \mathrm{mmHg})$ could decrease incidence and severity of shoulder pain compared with that associated with standard pressure $(>12 \mathrm{mmHg})$ during laparoscopic cholecystectomy (9). To date, most cases of laparoscopic surgery under regional anesthesia involve laparoscopic cholecystectomy by using low-pressure pneumoperitoneum. $(5,6,10)$. Only few reports 
regarding laparoscopic appendectomy have been published $(7,11)$. There are limited reports in the literature about laparoscopy under regional anesthesia performed in the field of gynecology $(12,13)$. Singh et al. studied 50 patients who underwent regional anesthesia-based laparoscopic surgery and reported that all laparoscopic surgeries were successfully performed under regional anesthesia using intrathecal fentanyl and low-pressure pneumoperitoneum, except in two cases (4\%). However, $42(84 \%)$ of the 50 patients underwent laparoscopic cholecystectomy and only $8(16 \%)$ underwent laparoscopic hysterectomy (12). Moawad et al. reported a case of total laparoscopic hysterectomy performed under epidural anesthesia in a patient who required regional anesthesia owing to previous side effects such as persistent dizziness and cognitive change over several days after deep sedation (13).

The high frequency of regional anesthesia in laparoscopic cholecystectomy can be attributed to the patient's posture. Laparoscopic cholecystectomy is performed in the reverse Trendelenburg position, whereas gynecological surgery is performed in the Trendelenburg position. Laparoscopic appendectomy is also performed in the Trendelenburg position, but there are lesser constraints for this procedure because the operation time in laparoscopic appendectomy is shorter than that in gynecologic surgery. Consequently, the Trendelenburg position and the use of $\mathrm{CO}_{2}$ gas are the two major factors that make application of regional anesthesia challenging in laparoscopic surgery. It is inevitable to assume a Trendelenburg position to secure surgical vision. The use of low-pressure pneumoperitoneum for regional anesthesia might be helpful for reducing the effects of this anesthesia on cardiovascular function, but the extent of reduction may be insufficient. Therefore, gasless laparoscopy can be an alternative to conventional laparoscopy that uses $\mathrm{CO}_{2}$ gas; the former technique can thus help avoid the potential negative effects of pneumoperitoneum such as cardiopulmonary dysfunction and shoulder pain. In this study, gasless SPA laparoscopy was performed to overcome these drawbacks with the patient being fully awake. We successfully performed gasless SPA laparoscopy in 30 patients under CSEA without any major complications and the need for converting to GA although one patient underwent laparotomy owing to severe pelvic adhesion.

Laparoscopic surgery under regional anesthesia can be potentially associated with the risk of a lack of adequate abdominal relaxation which obstructs the surgeon's ability to view the abdominal organ clearly and perform surgery. During laparoscopic surgery performed using $\mathrm{CO}_{2}$ gas under GA, muscle relaxation facilitates peritoneal insufflation and maintains an operative field (14). However, no problems related to the lack of abdominal relaxation have been reported in most of the articles, including this study. It is difficult to breathe in the presence of pneumoperitoneum and the viscera under regional anesthesia. The weight exerted by the omentum and viscera on the diaphragm might cause serious problems especially in obese patients. In severe cases of obesity, regional anesthesia should be administered with caution. Moreover, it may be difficult to secure surgical vision in obese patients, making the implementation of gasless laparoscopy also difficult. In this reason, obese patients were avoided showing that mean BMI $\left(\mathrm{kg} / \mathrm{m}^{2}\right)$ of patients included in this study was 21.9 in the CESA group and 23.3 in the GA group. BMI in the GA group is higher than in CSEA showing borderline significance $(p=0.054)$, which might be associated with lower mean setup time from umbilical skin incision to abdominal wall retraction in the CSEA group $(p=0.012)$. In addition, gasless laparoscopic surgery under CSEA was performed mainly in the latter part of the study, which is presumed to have contributed to the reduction of the mean setup time for the CSEA group.

The main problem of regional anesthesia in the Trendelenburg position is paralysis of the primary expiratory muscle. Placement of patients in the Trendelenburg position after administration of regional anesthesia can induce high spinal anesthesia, which can lead to shortness of breath and respiratory dysfunction. For maximal safety, an experienced anesthesiologist should attend and be prepared to convert to GA immediately in the event of complications. Several reports in the literature have suggested that the respiratory mechanism remains intact, and the respiratory muscle is unaffected under regional anesthesia. Ventilatory parameters and arterial blood gases without $\mathrm{CO}_{2}$ accumulation were maintained (15-17).

The success of laparoscopic surgery under regional anesthesia depends on appropriate management of pain and anxiety as well as adequate ventilation because the patient is fully awake. We presented music by ear phones to relieve patient anxiety during operation. Communication between an anesthesiologist and patients 
during surgery can help reduce patient anxiety. Although anxiety could be dealt with medications such as midazolam or propofol, the patient's consciousness was maintained in order to check for paralysis of the expiratory muscle to avoid the risk of aspiration in this study.

We successfully performed SPA gasless laparoscopy under CSEA without encountering any major complications. This approach can offer a valuable option to certain patients with poor cardiopulmonary function. Actually, this study included one patient who had history of old MI and coronary stent insertion (data not shown). CSEA is a safe and feasible option for non-obese patients undergoing gasless laparoscopic surgery in gynecologic field because gasless laparoscopy can help avoid the potential negative effects of $\mathrm{CO}_{2}$. The limitations of this study are associated with its retrospective design and small study population derived from a single institution. In the future, well-designed prospective, controlled studies investigating the physiologic advantages of gasless laparoscopy under CSEA are required to verify our findings.

\section{Disclosure of interests}

The authors declare that there are no conflicts of interest relevant to this study.

\section{Contribution of authorship}

JH and BK contributed to the conception and design of the study. JH and BK performed the clinical interventions. JH analyzed and interpreted the data. JH drafted the paper. All authors reviewed the article and approved the final version for publication.

\section{Details of ethical approval}

The study was reviewed and approved by the institutional review board of International St. Mary's Hospital, Catholic Kwandong University College of Medicine, Incheon, Republic of Korea (IS19RISI0043).

\section{Funding}

None

\section{Acknowledgements}

We would like to thank Dr. Young Soon Choi (Department of Anesthesiology and Pain medicine, International St. Mary's Hospital, Catholic Kwandong University College of Medicine, Incheon, Republic of Korea) for regional anesthesia and editorial comments.

\section{Figure Legends}

Figure 1. J shaped retractor installation. A J-shaped retractor was designed to fit in with attachment sites on the Thompson retractor. The Thompson retractor was mounted on the surgical table with an angled arm that fits in the joint of a rail clamp, and two J-shaped retractors are secured to the angled arm with articulation. Manually lift the J-shaped retractor is tightened to secure the working area.

\section{References}

1. Gerges FJ, Kanazi GE, Jabbour-Khoury SI. Anesthesia for laparoscopy: a review. J Clin Anesth. 2006;18(1):67-78.

2. Bajwa SJ, Kulshrestha A. Anaesthesia for laparoscopic surgery: General vs regional anaesthesia. J Minim Access Surg. 2016;12(1):4-9.

3. Collins LM, Vaghadia H. Regional anesthesia for laparoscopy. Anesthesiology clinics of North America. 2001;19(1):43-55.

4. Mazdisnian F, Palmieri A, Hakakha B, Hakakha M, Cambridge C, Lauria B. Office microlaparoscopy for female sterilization under local anesthesia. A cost and clinical analysis. J Reprod Med. 2002;47(2):97-100.

5. Longo MA, Cavalheiro BT, de Oliveira Filho GR. Laparoscopic cholecystectomy under neuraxial anesthesia compared with general anesthesia: Systematic review and meta-analyses. J Clin Anesth. 2017;41:48-54. 
6. Donmez T, Erdem VM, Uzman S, Yildirim D, Avaroglu H, Ferahman S, et al. Laparoscopic cholecystectomy under spinal-epidural anesthesia vs. general anaesthesia: a prospective randomised study. Ann Surg Treat Res. 2017;92(3):136-42.

7. Uzman S, Donmez T, Erdem VM, Hut A, Yildirim D, Akinci M. Combined spinal-epidural anesthesia in laparoscopic appendectomy: a prospective feasibility study. Ann Surg Treat Res. 2017;92(4):208-13.

8. Alexander JI. Pain after laparoscopy. Br J Anaesth. 1997;79(3):369-78.

9. Sarli L, Costi R, Sansebastiano G, Trivelli M, Roncoroni L. Prospective randomized trial of low-pressure pneumoperitoneum for reduction of shoulder-tip pain following laparoscopy. The British journal of surgery. 2000;87(9):1161-5.

10. Mehta N, Dar MR, Sharma S, Mehta KS. Thoracic combined spinal epidural anesthesia for laparoscopic cholecystectomy: A feasibility study. J Anaesthesiol Clin Pharmacol. 2016;32(2):224-8.

11. Mane RS, Patil MC, Kedareshvara KS, Sanikop CS. Combined spinal epidural anesthesia for laparoscopic appendectomy in adults: A case series. Saudi J Anaesth. 2012;6(1):27-30.

12. Singh RK, Saini AM, Goel N, Bisht D, Seth A. Major laparoscopic surgery under regional anesthesia: A prospective feasibility study. Med J Armed Forces India. 2015;71(2):126-31.

13. Moawad NS, Santamaria Flores E, Le-Wendling L, Sumner MT, Enneking FK. Total Laparoscopic Hysterectomy Under Regional Anesthesia. Obstet Gynecol. 2018;131(6):1008-10.

14. Staehr-Rye AK, Rasmussen LS, Rosenberg J, Juul P, Lindekaer AL, Riber C, et al. Surgical space conditions during low-pressure laparoscopic cholecystectomy with deep versus moderate neuromuscular blockade: a randomized clinical study. Anesth Analg. 2014;119(5):1084-92.

15. Nishio I, Noguchi J, Konishi M, Ochiai R, Takeda J, Fukushima K. [The effects of anesthetic techniques and insufflating gases on ventilation during laparoscopy]. Masui. 1993;42(6):862-6.

16. Ciofolo MJ, Clergue F, Seebacher J, Lefebvre G, Viars P. Ventilatory effects of laparoscopy under epidural anesthesia. Anesth Analg. 1990;70(4):357-61.

17. van Zundert AA, Stultiens G, Jakimowicz JJ, Peek D, van der Ham WG, Korsten HH, et al. Laparoscopic cholecystectomy under segmental thoracic spinal anaesthesia: a feasibility study. Br J Anaesth. 2007;98(5):682-6.

Table 1. Clinical characteristics of patients who underwent gasless SPA laparoscopy $(\mathrm{N}=90)$

Variables

Ages (year: mean $\pm \mathrm{SD}$ )

Body weight $(\mathrm{kg}$, mean $\pm \mathrm{SD})$

Height $(\mathrm{cm}$, mean $\pm \mathrm{SD})$

Body mass index $\left(\mathrm{kg} / \mathrm{m}^{2}:\right.$ mean $\left.\pm \mathrm{SD}\right)$

Parity, number (\%)

None

One

Two

More than three

Previous abdominal surgery, Number (\%)

None

One time

Two times

More than three times

Previous abdominal surgery type (\%)
Table 1. Clinical character Under CESA $(\mathrm{N}=30)$

$41.4 \pm 11.3$

$55.6 \pm 5.5$

$159.6 \pm 5.4$

$21.9 \pm 2.8$

$10(33.3)$

$6(20)$

$13(43.3)$

$1(3.3)$

$16(53.3)$

$7(23.3)$

$4(13.3)$

3 (10) 
Table 1. Clinical characteristics of patients who underwent gasless SPA laparoscopy $(\mathrm{N}=90)$

Cesarean section

Appendectomy

Laparoscopic operation

Myomectomy

Liver donor
Table 1. Clinical character $10(33.3)$

3 (10)

2 (6.6)

0

$1(3.3)$

* Abbreviation: SPA, single port approach; SD, Standard deviation; CESA; Combined epidural spinal anesthesia; GA, General anesthesia

Table 2. Characteristics of Combined spinal and epidural anesthesia $(\mathrm{N}=30)$

Characteristics

Conversion to general anesthesia

Paresthesia from spinal needle insertion

CSE block

\section{L $3-4$}

L 4-5

Peak sensory level

T 2

T 3

$\mathrm{T} 4$

Intraoperative adverse event

Anxiety requiring midazolam

Nausea/vomiting requiring anti-emetics

Hypotension (Systolic blood pressure $<90 \mathrm{mmHg}^{\mathrm{a}}$ )

Shoulder pain or abdominal discomfort requiring analgesics

Bradycardia (Heart rate $<50$ rate per minute ${ }^{\mathrm{a}}$ )

Respiratory discomfort
Table 2. Characteristics of Combined sp Gasless SPA laparoscopy, N (\%) 0

0

$29(96.7)$

$1(3.3)$

$1(3.3)$

$5(16.7)$

$24(80)$

$12(40)$

$6(20)$

$5(16.7)$

$4(13.3)$

$1(3.3)$

0

a; occurs at least once during operation

Table 3. Surgical Outcomes of gasless SPA laparoscopy $(\mathrm{N}=90)$. Table 3. Surgical Outcomes of gasless SPA laparoscopy

Variables

Type of surgery, Number (\%)

Adnexal surgery ${ }^{\mathrm{a}}$

hysterectomy

Myomectomy

Specific surgical finding, Number (\%)

Severe pelvic adhesion

Mass rupture

Torsion

From incision to setup time (minute, mean $\pm \mathrm{SD}$ )

Operation Time (minute, mean $\pm \mathrm{SD}$ )

Estimated Blood loss (ml, mean \pm SD)

Hospital stay (day, mean \pm SD)

Laparotomy conversion, Number (\%)

Use of additional trocar (two port), Number (\%)

Major complications ${ }^{\mathrm{b}}$
Under CESA $(\mathrm{N}=30)$

$18(60)$

$6(20)$

$6(20)$

$3(10)$

$1(3.3)$

1 (3.3)

$6.8 \pm 1.8$

$68.9 \pm 27$

$96.7 \pm 84$

$4.5 \pm 0.6$

1 (3.3)

$1(3.3)$

None 
a: Include urologic, bowel and vessel injuries

b: Include cystectomy, salpingectomy and salpingo-oophorectomy

* Abbreviation: SPA, single port approach; SD, Standard deviation; CESA, Combined epidural spinal anesthesia; G/A, General anesthesia

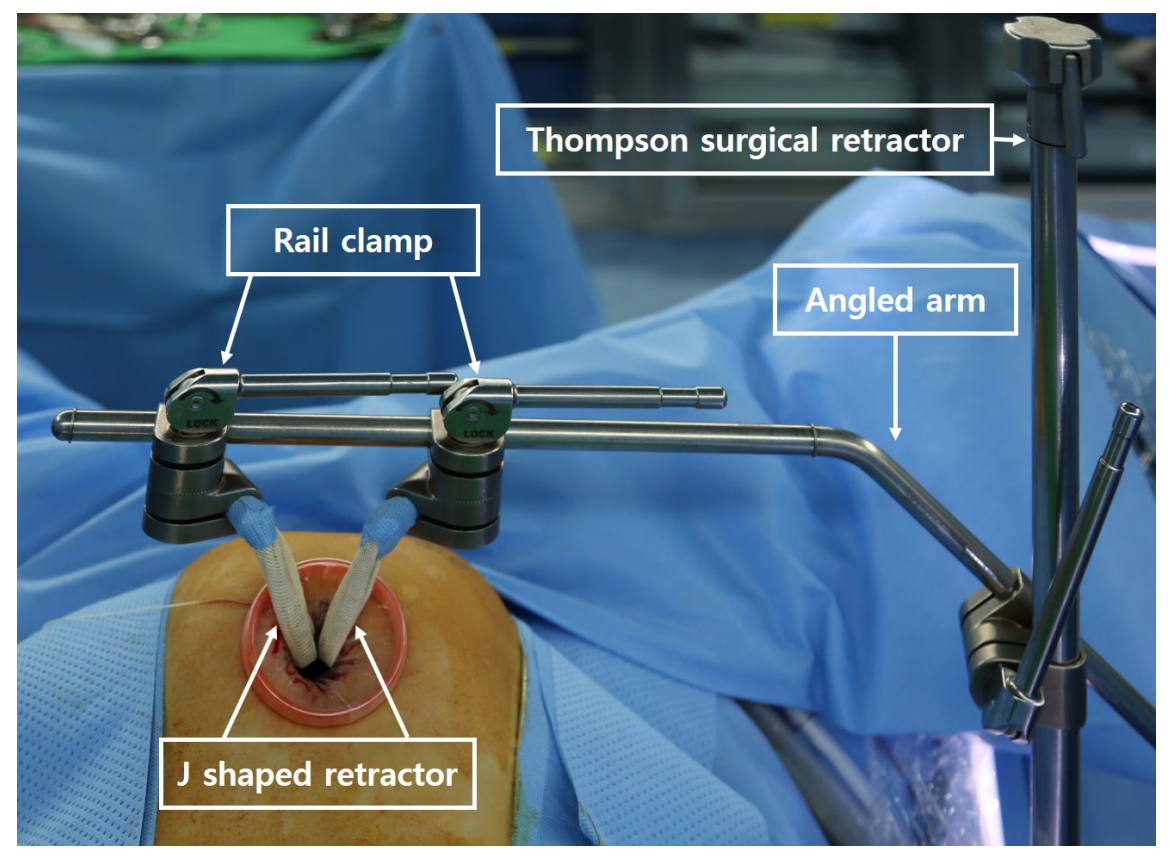

